\title{
Predictors of Medication-Assisted Treatment Initiation for Opioid Use Disorder in an Interdisciplinary Primary Care Model
}

\author{
Rebecca E. Cantone, MD, Brian Garvey, MD, MPH, Allison O'Neill, MPH, \\ Joan Fleishman, PsyD, Deborah Cohen, PhD, John Muench, MD, MPH, and \\ Steffani R. Bailey, PhD
}

Introduction: Medication-assisted treatment (MAT) for opioid use disorder (OUD) is underused in primary care. Little is known about patient demographics associated with MAT initiation, particularly among models with an interdisciplinary approach, including behavioral health integration. We hypothesize few disparities in MAT initiation by patient characteristics after implementing this model for OUD.

Methods: Electronic health record data were used to identify adults with $\geq 1$ primary care visit in 1 of 2 study clinics in a Pacific Northwest academic health system between September 1, 2015 and August 31, $2017(\mathrm{n}=23,372)$. Rates of documented OUD diagnosis were calculated. Multivariate logistic regression estimated odds ratios of MAT initiation, defined as $\geq 1$ electronic health record order for buprenorphine or naltrexone, by patient covariates.

Results: Seven percent of the study sample had an OUD diagnosis. Of those patients, $32 \%$ had $\geq 1$ MAT order. Patients with documented psychiatric diagnoses or tobacco use had higher odds of initiating MAT (odds ratio $[\mathrm{OR}]=1.62, P=.0003 ; 0 R=2.46, P<.0001$, respectively). Uninsured, Medicaid, and Medicare patients had lower odds than those commercially insured $(\mathrm{OR}=0.53,0.38$, and 0.31 , respectively; $\boldsymbol{P}<.0001)$. Patients who were older, of a race/ethnicity other than non-Hispanic white, had documented diabetes, and had documented asthma or chronic obstructive pulmonary disease showed lower odds of initiation.

Discussion: MAT initiation varied by patient characteristics, including disparities by insurance coverage and race/ethnicity. The addition of behavioral health did not eliminate disparities in care, but higher odds of initiation among those with a documented psychiatric diagnosis may suggest this model reaches some vulnerable populations. Additional research is needed to further examine these findings. (J Am Board Fam Med 2019;32:724-731.)

Keywords: Addiction Medicine, Health Care Disparities, Mental Health, Northwestern United States, Opioid-Related Disorders, Primary Health Care

Opioid use disorder (OUD) is a significant public health concern in the United States. From 1999 to 2016, drug overdoses resulted in 632,331 deaths in the United States, including 351,630 opioid over-

This article was externally peer reviewed.

Submitted 14 January 2019; revised 5 May 2019; accepted 6 May 2019.

From the Department of Family Medicine, Oregon Health \& Science University, Portland, OR (REC, BG, JF, DC, JM, SRB); OCHIN, Inc. Portland, OR (AO, JM).

Funding: NIDA awards K23-DA037453, UG1DA015815, and R01DA046468; and internal funding from the Department of Family Medicine, Oregon Health \& Science University.

Conflict of interest: none declared. dose deaths. ${ }^{1}$ Drug overdose is now the leading cause of accidental death in the United States, ${ }^{2}$ with an increase especially with synthetic opioids through 2016. ${ }^{3}$ In addition to the health-related consequences of OUD, estimated attributable annual health care costs range from $\$ 10,000$ to $\$ 20,000$ per patient. $^{4-6}$

Medication-assisted treatment (MAT) for OUD, including buprenorphine and naltrexone, has positive

Corresponding author: Rebecca E. Cantone, MD, Department of Family Medicine, Oregon Health \& Science University, Portland, OR 97239 (E-mail: cantone@ohsu.edu). 
impacts on treatment retention ${ }^{7-10}$ and is positively associated with an increased quality of life. ${ }^{11} \mathrm{Bu}-$ prenorphine is an alternative to methadone that can be used in primary care, ${ }^{10}$ is an effective treatment for OUD compared with abstinence-only treatment models, ${ }^{10,12,13}$ and is currently underused. ${ }^{14,15}$ Limited research has shown disparities in MAT initiation in primary care settings by age, ${ }^{14,16}$ race/ethnicity, ${ }^{16}$ and health insurance coverage. ${ }^{14,16}$ Findings regarding the relationships between MAT initiation and psychiatric and physical comorbidities are mixed and seem to vary depending on the specific comorbidity. ${ }^{14}$ However, mental and physical health conditions are known to be common in patients with OUD, ${ }^{17}$ and patients with mental illness have higher rates of substance use. ${ }^{18}$ This strong association between substance use and psychiatric comorbidities ${ }^{19}$ can make initiation and maintenance in MAT programs more difficult in this population. ${ }^{20}$

There are multiple models of MAT that exist in primary care settings, with differing levels of behavioral health integration. ${ }^{21,22}$ Before implementing the interdisciplinary MAT model studied, patients with OUD could see behavioral health specialists at their primary care clinic as part of their OUD treatment, but it was not a standardized component of the MAT program. The model implemented in the study clinics used for this analysis was adapted from the Family Health Center of Worcester model in the state of Massachusetts. It used buprenorphine-containing medications and naltrexone, and added masters-level behavioral health providers to the primary care team to work with patients on improving coping skills, relapse prevention, and resilience strategies, with the goal of holistically addressing the complex psychosocial needs of patients with OUD. ${ }^{23}$ In both clinics, behavioral health providers had specific training in working with patients with substance use disorders (SUDs). Medical and behavioral health providers at these clinics also trained in and practice traumainformed care and a harm reduction approach to treatment. The interdisciplinary model expanded the role of behavioral health by encouraging continuity visits and regular assessments throughout engagement in the MAT program, which also supported providers in diagnosing and treating SUDs. The behavioral health team worked in collaboration with a registered nurse (RN) who focused on care management and monitored the frequency of visits. MAT was initiated with an assessment by a registered nurse, behavioral health provider, and medical provider to determine if the program was an appropriate level of care for the patient and if a prescription for a buprenorphine-containing medication or naltrexone was appropriate. Patients who entered this MAT model started with frequent visits (up to several times a week) and could progress to less frequent visits (up to every 60 days) as they engaged in their recovery and increased in stability. ${ }^{23}$

Little is known about the characteristics of patients who initiate MAT in primary care programs with robust behavioral health support. Our study examined the prevalence of OUD diagnosis and patient characteristics (e.g., demographics and medical comorbidities) associated with the initiation of MAT (buprenorphine or naltrexone) in the 2 primary care clinics that implemented the interdisciplinary program described above with the aim to engage underserved patients with OUD in treatment. With a more holistic approach to MAT care in these clinics that included education on reducing stigma around patients with OUD and training in trauma informed care, we postulated that that patients who initiated MAT would have similar characteristics to those who did not initiate MAT. We also hypothesized that we would see higher odds of MAT initiation among Medicaidinsured individuals, as there may be fewer alternative OUD treatment options covered by that insurance in the geographic areas these clinics serve. This study aimed to better understand who engaged with the MAT program to help lay groundwork for an expanded line of inquiry to evaluate and improve MAT models with significant behavioral health involvement.

\section{Methods}

\section{Study Design}

This was a retrospective observational study.

\section{Setting}

This study was conducted in 2 academic primary care clinics within the same health care system in the Pacific Northwest. One practice was a Rural Health Clinic and the other was a Federally Qualified Health Center. Both of these primary care clinics received supplemental payments to subsidize underserved care because of their Rural Health Clinic or Federally Qualified Health Center status, had an integrated behavioral 
health model and trauma informed care training, and implemented the interdisciplinary MAT model described above in late 2015 to early 2016 to improve the care of patients with SUDs, specifically OUD. These clinics were selected for the study as they shared the same electronic health record (EHR), both followed a similar treatment philosophy for addiction, and shared clinical protocols and staffing models.

Study data were extracted from structured fields of the clinics' EHRs. Both clinics used an EHR supported by OCHIN, a nonprofit health information technology organization which provides a single, linked instance (each patient has a single identification number and medical record shared across every clinic in the network) of the Epic EHR. ${ }^{24}$

This study was approved by our institution's Institutional Review Board.

\section{Study Population}

To examine the prevalence of documented OUD, our denominator included adults aged 18 years and older at the start of the study who had at least 1 primary care visit to either of the 2 clinics between September 1, 2015 and August 31, 2017. To examine predictors of MAT initiation, we evaluated a subset of patients who met the above criteria and also had a documented diagnosis of OUD.

\section{Variables}

Opioid use disorder

A patient was identified as having an OUD diagnosis if any of the following International Classification of Diseases, 9th and 10th Revision (ICD-9/ 10) codes were documented in the problem list or an encounter list during the study period: ICD-9: 304.00, 304.01, 304.02; ICD-10: F11.1 **; F11.2**.

\section{MAT initiation}

We extracted MAT orders during the study period, defined as an electronic prescription order in the EHR for a medication containing buprenorphine or injectable naltrexone. MAT initiation was dichotomized (yes vs no); yes represents that a patient had $\geq 1$ order for a form of buprenorphine, buprenorphine-naltrexone, or injectable naltrexone (all brand names included). To enter the program, patients can be referred by a medical or behavioral health provider or be self-referred. Patients complete an in-person assessment by a medical or behavioral health pro- vider, inclusive of the goals and expectations of both the patient and the program.

\section{Patient characteristics}

We extracted discrete data from the EHR for the following characteristics thought to be associated with SUD treatment ${ }^{16}$ : age, sex, race/ethnicity by self-identification, health insurance coverage, smoking status as identified in a discrete data field in the vital signs or social history, and medical and psychiatric comorbidities as identified via ICD9/10 codes: hypertension, diabetes, asthma/chronic obstructive pulmonary disease (COPD), coronary artery disease, dyslipidemia, human immunodeficiency virus, hepatitis $\mathrm{C}$, cancer, psychiatric disorders including anxiety disorders, posttraumatic stress disorder, schizophrenia and other psychotic disorders, depressive disorders, and bipolar disorder. We also identified whether a patient's clinic was urban or rural.

\section{Analysis}

We first calculated the prevalence of OUD diagnosis among all patients meeting the study inclusion criteria. We then described the characteristics of the subset of patients with OUD, total, and by MAT initiation. Logistic regression was performed with $\geq 1$ MAT order (vs none) as the dependent variable and the patient characteristics as the independent variables. Univariate logistic regression modeling was performed to determine the statistical significance of each variable with MAT initiation. Race/ethnicity categories other than non-Hispanic white were collapsed due to low numbers of patients in the separate race/ ethnicity categories.

Age, sex, race/ethnicity, and all other characteristics noted above that were significant at the $P<$ .05 level in the univariate analyses were included in the multivariate logistic regression model. All statistical analyses were conducted in 2017 using SAS Enterprise Guide, version 7.13 (SAS Institute Inc., Cary, NC). All statistical tests were two-sided and significance was defined as a $P<.05$.

\section{Results}

\section{Opioid Use Disorder Diagnosis}

Of the 23,372 unique individuals seen at 1 of the 2 clinic sites, 7\% ( $\mathrm{n}=1638$ patients) had a documented OUD diagnosis during the study period. 
Table 1. Characteristics of Patients with Opioid Use Disorder, Total, and by Medication-Assisted Treatment Initiation Versus No MAT Initiation

\begin{tabular}{|c|c|c|c|c|}
\hline & Total & MAT Initiation & No MAT Initiation & \\
\hline Patient Characteristics & N (\%) & $\mathrm{N}(\%)$ & N (\%) & $P$ Value \\
\hline Total & 1638 & $542(33.1)$ & $1096(66.9)$ & \\
\hline Urban/rural & & & & .5776 \\
\hline Urban & $1222(74.6)$ & $404(74.5)$ & $818(74.6)$ & \\
\hline Rural & $416(25.4)$ & $138(25.5)$ & $278(25.4)$ & \\
\hline Sex & & & & .2946 \\
\hline Male & $658(40.2)$ & $208(38.4)$ & $450(41.1)$ & \\
\hline Female & $980(59.8)$ & $334(61.6)$ & $646(58.9)$ & \\
\hline Age as of date of first MAT prescription & & & & $<.0001$ \\
\hline 18 to 29 & $332(20.3)$ & $149(27.5)$ & NA & \\
\hline 30 to 49 & $791(48.3)$ & $282(52.0)$ & NA & \\
\hline $50+$ & $515(31.4)$ & $111(20.5)$ & NA & \\
\hline Race/Ethnicity (detail) & & & & .5064 \\
\hline Hispanic & $46(2.8)$ & $16(2.9)$ & $30(2.7)$ & \\
\hline NH white & $1358(82.9)$ & $457(84.3)$ & $901(82.2)$ & \\
\hline NH other & $52(3.2)$ & $12(2.2)$ & $40(3.6)$ & \\
\hline NH black & $94(5.7)$ & $24(4.4)$ & $70(6.4)$ & \\
\hline Unknown & $88(5.4)$ & $33(6.1)$ & $55(5.0)$ & \\
\hline Race/Ethnicity (for analysis) & & & & .0106 \\
\hline NH white & $1358(82.9)$ & $457(84.3)$ & $901(82.2)$ & \\
\hline Other & $280(17.1)$ & $85(15.7)$ & $195(17.8)$ & \\
\hline Insurance coverage at majority of visits in study year & & & & $<.0001$ \\
\hline Commercial & $128(7.8)$ & $65(11.9)$ & $63(5.79)$ & \\
\hline Medicaid & $977(59.6)$ & $315(58.1)$ & $662(60.9)$ & \\
\hline Medicare & $222(13.6)$ & $45(8.3)$ & $177(16.3)$ & \\
\hline Self-pay & $302(18.4)$ & $116(21.4)$ & $186(17.1)$ & \\
\hline Other & $<10$ & $<10$ & $<10$ & \\
\hline \multicolumn{5}{|l|}{ Comorbidities } \\
\hline Hypertension & $463(28.3)$ & $110(20.3)$ & $353(32.2)$ & .0215 \\
\hline Diabetes & $166(10.1)$ & $27(4.9)$ & $139(12.7)$ & .0011 \\
\hline Asthma/COPD & $360(21.9)$ & $86(15.9)$ & $274(25.0)$ & $<.0001$ \\
\hline Coronary artery disease & $62(3.8)$ & $<10$ & $55(5.02)$ & .0020 \\
\hline Lipid disorder & $251(15.3)$ & $50(9.2)$ & $201(18.3)$ & $<.0001$ \\
\hline Cancer & $130(7.9)$ & $27(4.9)$ & $103(9.4)$ & .0029 \\
\hline HIV & $<10$ & 0 & $<10$ & .5380 \\
\hline Hepatitis C & $174(10.6)$ & $50(9.2)$ & $124(11.3)$ & .0331 \\
\hline Psychiatric disorder* & $1204(73.5)$ & $419(77.3)$ & $785(71.6)$ & .0106 \\
\hline Current tobacco use at $\geq 1$ visit & $948(57.9)$ & $386(71.2)$ & $562(51.3)$ & $<.0001$ \\
\hline
\end{tabular}

NA, Not applicable; NH, Non-Hispanic; COPD, chronic obstructive pulmonary disease; HIV, human immunodeficiency virus; MAT, Medication-Assisted Treatment.

*Psychiatric disorders included anxiety disorders, posttraumatic stress disorder, schizophrenia and other psychotic disorders, depressive disorders, and bipolar disorder.

Of those with an OUD diagnosis, the majority were female (59.8\%), $68.6 \%$ were between the ages of 18 and $49,82.9 \%$ identified as non-Hispanic white, and $59.6 \%$ were Medicaid insured. Over $70 \%(73.5 \%)$ had a comorbid psychiatric disorder and $57.9 \%$ were current smokers. Table 1 shows the characteristics of patients with OUD in total and by MAT initiation.

\section{MAT Initiation and Predictors}

Of the 1638 individuals with documented OUD, $33 \%$ had $\geq 1$ MAT order $(\mathrm{n}=542)$. The univariate 
Table 2. Adjusted Odds Ratios of Medication-Assisted Treatment Initiation for Opioid Use Disorder by Patient Characteristics $(\mathrm{n}=1638) *$

\begin{tabular}{|c|c|c|c|}
\hline Characteristic & Odds Ratio & 95\% Confidence Interval & $P$ Value \\
\hline \multicolumn{4}{|l|}{ Sex } \\
\hline Male & Ref & & \\
\hline Female (ref: male) & 1.04 & $0.82,1.30$ & 0.7719 \\
\hline Age as of date of first MAT prescription & & & 0.0030 \\
\hline 18 to 29 & Ref & & \\
\hline 30 to 49 & 0.77 & $0.58,1.01$ & \\
\hline $50+$ & 0.54 & $0.38,0.77$ & \\
\hline Race/Ethnicity & & & 0.7528 \\
\hline Non-Hispanic white & Ref & & \\
\hline Other & 0.95 & $0.71,1.28$ & \\
\hline Insurance coverage at majority of visits in study period & & & $<0.0001$ \\
\hline Commercial & Ref & & \\
\hline Medicaid & 0.38 & $0.26,0.57$ & \\
\hline Medicare & 0.31 & $0.18,0.53$ & \\
\hline Self-pay & 0.53 & $0.34,0.83$ & \\
\hline \multicolumn{4}{|l|}{ Comorbidities $^{\dagger}$} \\
\hline Hypertension & 0.89 & $0.67,1.19$ & 0.4495 \\
\hline Diabetes & 0.56 & $0.35,0.89$ & 0.0135 \\
\hline Asthma/COPD & 0.70 & $0.53,0.94$ & 0.0186 \\
\hline Coronary artery disease & 0.54 & $0.24,1.26$ & 0.1537 \\
\hline Lipid disorder & 0.71 & $0.49,1.03$ & 0.0714 \\
\hline Cancer & 0.68 & $0.43,1.09$ & 0.1084 \\
\hline Hepatitis C & 0.73 & $0.51,1.05$ & 0.0934 \\
\hline Psychiatric disorder $^{\ddagger}$ & 1.62 & $1.25,2.10$ & 0.0003 \\
\hline Current tobacco use at $\geq 1$ visit & 2.46 & $1.95,3.12$ & $<0.0001$ \\
\hline
\end{tabular}

COPD, chronic obstructive pulmonary disease. MAT, medication-assisted treatment.

*Bolded values denote statistical significance at $P<.05$.

${ }^{\dagger}$ Reference for each comorbid condition is the absence of the condition.

${ }^{\ddagger}$ Psychiatric disorders included anxiety disorders, posttraumatic stress disorder, schizophrenia and other psychotic disorders, depressive disorders, and bipolar disorder.

analysis found no significant differences in MAT initiation by sex, human immunodeficiency virus status, clinic type (rural or urban), coronary artery disease, lipid disorder, cancer, or hepatitis C. Table 2 shows the adjusted odds ratios (aOR) and 95\% confidence intervals (CIs) for the patient characteristics included in the multivariate logistic regression model. Patients with public insurance $(\mathrm{aOR}=$ $0.38,95 \%$ CI: 0.26 to 0.57 for Medicaid; aOR = $0.31,95 \%$ CI: 0.18 to 0.53 for Medicare) or selfpay (aOR $=0.53,95 \%$ CI: 0.34 to 0.83 ) had lower odds of having a MAT order than patients who had commercial insurance. Patients $>50$ years of age had lower odds of MAT initiation than patients who were 18 to 29 years of age $(\mathrm{aOR}=0.54,95 \%$ CI: 0.38 to 0.77 ). Patients with a documented diagnosis of diabetes and asthma/COPD also had lower odds than those without these diagnoses $(\mathrm{aOR}=0.56,95 \%$ CI: 0.35 to 0.89 ; $\mathrm{aOR}=0.70$, 95\% CI: 0.53 to 0.94 , respectively). Patients who reported current tobacco use had over 2 times higher odds of initiating MAT compared with patients who were not tobacco users $(\mathrm{aOR}=2.46$, 95\% CI: 1.95 to 3.12 ) and those with a psychiatric diagnosis had $62 \%$ increased odds of initiating MAT compared with those without a documented psychiatric disorder $(\mathrm{aOR}=1.62,95 \% \mathrm{CI}: 1.25$ to 2.10).

\section{Discussion}

This study examined the prevalence of OUD diagnosis and patient characteristics associated with MAT initiation in 2 primary care clinics after im- 
plementing a similar practice-based improvement model of MAT that included nurse care management and robust behavioral health involvement. Although the prevalence of OUD among patients seen in primary care settings is unknown, the Centers for Disease Control and Prevention reported a prevalence in 2016 (ages 12 years and older) at $4.4 \%$ for opioid misuse in the past year and $0.8 \%$ for OUD in the past year. ${ }^{25}$ We observed a higher prevalence in our clinics of $7 \%$. This was also higher than in a previous study among safety-net populations ${ }^{14}$ and could be due to decreased stigma-inducing behaviors from clinical teams facilitated by efforts to view OUD as a medical disease, trainings on trauma informed care and harm reduction, and recruitment of medical and behavioral health providers dedicated to helping patients with OUD. Although many medical providers practice abstinence-only methods, other medical providers in the clinic could prescribe buprenorphine or naltrexone if the patient sought that care. We believe that the patient-level "word of mouth" recommendation to others may have contributed to increased interest in our program from both our own clinic patients and the community at large, which may be due to the robust behavioral health component, clinician treatment philosophy, or limited supportive treatment options elsewhere in these communities.

Patients with OUD who initiated MAT had higher odds of having a documented psychiatric diagnosis and current tobacco use status than patients with OUD who did not initiate MAT. Several explanations for these findings are possible. First, the integrated behavioral health model may have increased patients' willingness to initiate MAT, due to the ability to address both the psychiatric diagnosis and OUD within the primary care setting and with the same providers. A second possibility is that prior engagement with behavioral health may have facilitated MAT initiation, increasing the likelihood that patients attend and complete the initial visit through supportive actions by the behavioral health team. Finally, it is plausible that patients who initiate MAT are more likely to be screened for psychiatric disorders during a behavioral health intake for the MAT program than with a routine medical visit. As behavioral health providers often screen for both mental health conditions and substance-induced disorders, including tobacco use, this may lead to a misclas- sification bias, with patients more likely to have a psychiatric diagnoses or SUD such as tobacco use formally documented. Although our MAT program increased the complexity of treatment with more frequent visits, those with psychiatric diagnoses still initiated treatment. This is an important finding as patients with SUD often have comorbid psychiatric diagnoses ${ }^{18,19,26}$ and often face multiple barriers to accessing care; however, these patients still initiated treatment in our program.

Contrary to our hypotheses, the results identified potential disparities in access to care for certain vulnerable and high-risk populations. We found differences in MAT initiation by health insurance coverage, age, and race/ethnicity, similar to prior studies. ${ }^{14,16}$ Those with public insurance or no insurance had lower odds of MAT receipt than patients with commercial insurance. Despite public insurance coverage of these medications and office visits, disparities continued to exist.

We also found lower odds of MAT initiation among older patients than their younger counterparts, as well as lower odds of MAT initiation among patients of a race/ethnicity other than nonHispanic white. These findings suggest the need for further development of MAT models to reach all demographic groups and may need to include further sensitivity to generational or cultural needs.

We found few significant differences in MAT initiation by medical comorbidities; however, patients with diabetes and asthma or COPD had lower odds of initiating MAT than those without these documented diagnoses. Odds ratios were less than 1 for all medical comorbidities, albeit not statistically significant. There are possible explanations for this finding, such as time constraints and a focus on medical issues during a primary care office visit among patients with chronic medical conditions, limiting medical providers' ability to address SUD. Patients with chronic medical conditions that require frequent visits may decline OUD treatment that requires additional clinical encounters. Finally, patients may choose different clinics or systems for SUD treatment.

This study had a number of important limitations. The cross-sectional design precludes the inference of a causal relationship between implementation of the interdisciplinary model of care and differing odds of MAT initiation by patient demographics. We did not have access to data to compare demographics of patients who initiated MAT 
pre- and post-implementation of the integrated MAT model.

We also were unable to examine whether prior engagement in behavioral health services was associated with subsequent MAT initiation in the program. That might provide evidence that engagement with behavioral health providers can be leveraged to treat patients with OUD and may be an independent factor in the success of our program. Further studies should examine if the use of behavioral health services has an effect on MAT initiation.

In addition, although the prevalence of OUD was around $7 \%$, it is possible that some patients met criteria for OUD but did not have a formal chart diagnosis. Despite the clinics' initiatives to increase SUD screening, there remains a likelihood of underreporting of patients with OUD. This could be due to provider gaps in the knowledge of diagnostic criteria for patients on chronic prescription or other opioids, failure to add the diagnosis to the problem list, or use of an ICD-9/10 code not examined in this study (such as polysubstance use). We also were unable to ascertain if patients with OUD did not initiate MAT treatment because providers did not offer it, the patient declined treatment, or the patient was referred to more intensive treatment outside of the primary care setting. Although insurance coverage does not affect provider reimbursement, we cannot rule out a treatment bias based on the insurance viewable by providers.

Finally, our patient population was mostly nonHispanic white, limiting our ability to examine differences in MAT initiation by other races and ethnicities. Future research is needed in populations that are more diverse to further our understanding of MAT treatment initiation among racial and ethnic minorities.

\section{Conclusion}

MAT initiation varied by patient characteristics with higher odds of MAT initiation among patients with documented psychiatric diagnoses and tobacco use, suggesting increased reach of these vulnerable populations; however, there were disparities in MAT initiation by insurance and race/ ethnicity. The persistence of these disparities is concerning from a health equity standpoint. Future research is needed to determine the etiology of these differences to inform policies, the design of
MAT treatment models, and practice-based improvements in efforts to reach historically underserved populations.

Dennis McCarty, $\mathrm{PhD}$, for contributing to the study concept and for editing previous versions of this manuscript; and Philip Bolduc, MD, FHCW, OBOT Program Director at the Family Health Center of Worcester for sharing his program documents leading to the initial conception of this treatment model. We also acknowledge the MAT working group at the Oregon Health \& Science University Department of Family Medicine.

To see this article online, please go to: http://jabfm.org/content/ 32/5/724. full.

\section{References}

1. Seth P, Scholl L, Rudd RA, Bacon S. Overdose deaths involving opioids, cocaine, and psychostimulants-United States, 2015-2016. MMWR Morb Mortal Wkly Rep 2018;67:349-58.

2. Murphy SL, Xu J, Kochanek KD, Curtin SC, Arias E. Deaths: Final data for 2015. Natl Vital Stat Rep 2017;66:1-122.

3. Hedegaard H, Warner M, Miniño AM. Drug overdose deaths in the United States, 1999-2016. NCHS Data Brief 2017;294:1-8.

4. Kirson NY, Scarpati LM, Enloe CJ, Dincer AP, Birnbaum HG, Mayne TJ. The economic burden of opioid abuse: updated findings. J Manag Care Spec Pharm 2017;23:427-45.

5. Meyer R, Patel AM, Rattana SK, Quock TP, Mody $\mathrm{SH}$. Prescription opioid abuse: a literature review of the clinical and economic burden in the United States. Popul Health Manag 2014;17:372-87.

6. Rice JB, Kirson NY, Shei A, et al. Estimating the costs of opioid abuse and dependence from an employer perspective: a retrospective analysis using administrative claims data. Appl Health Econ Health Policy 2014;12:435-46.

7. Thomas CP, Fullerton CA, Kim M, et al. Medication-assisted treatment with buprenorphine: assessing the evidence. Psychiatr Serv 2014;65:158-70.

8. Timko C, Schultz NR, Cucciare MA, Vittorio L, Garrison-Diehn C. Retention in medication-assisted treatment for opiate dependence: a systematic review. J Addict Dis 2016;35:22-35.

9. Connery HS. Medication-assisted treatment of opioid use disorder: review of the evidence and future directions. Harv Rev Psychiatry 2015;23:63-75.

10. Mattick RP, Breen C, Kimber J, Davoli M. Buprenorphine maintenance versus placebo or methadone maintenance for opioid dependence. Cochrane Database Syst Rev 2014;2:CD002207.

11. Mitchell SG, Gryczynski J, Schwartz RP, et al. Changes in quality of life following buprenorphine treatment: relationship with treatment retention and illicit opioid use. J Psychoactive Drugs 2015; 47:149-57. 
12. Vestal C. Report from the field: In fighting an opioid epidemic, medication-assisted treatment is effective but underused. Health Aff 2016;35:1052-7.

13. Srivastava A, Kahan M, Nader M. Primary care management of opioid use disorders. Can Fam Physician 2017;63:200-5.

14. Rieckmann T, Muench J, McBurnie MA, et al. Medication-assisted treatment for substance use disorders within a national community health center research network. Subst Abus 2016;37:625-34.

15. Hill RR. Medication-assisted treatment should be part of every family physician's practice: no. Ann Fam Med 2017;15:310-2.

16. Rieckmann TR, Gideonse N, Risser A, DeVoe JE, Abraham AJ. Treating opioid dependence with buprenorphine in the safety net: critical learning from clinical data. J Behav Heal Serv Res 2017;44:351-63.

17. Lagisetty PA, Maust D, Heisler M, Bohnert A. Physical and mental health comorbidities associated with primary care visits for substance use disorders. J Addict Med 2017;11:161-2.

18. Substance Abuse and Mental Health Services Administration. Results from the 2013 National Survey on Drug Use and Health: Mental Health Findings, NSDUH Series H-49, HHS Publication No. (SMA) 14-4887. Rockville, MD: Substance Abuse and Mental Health Services Administration. Available from: https:/www.samhsa.gov/data/sites/default/files/ NSDUHmhfr2013/NSDUHmhfr2013.pdf. Published 2014. Accessed January 11, 2019.

19. Lai HMX, Cleary M, Sitharthan T, Hunt GE. Prevalence of comorbid substance use, anxiety and mood disorders in epidemiological surveys, 1990-2014: a systematic review and meta-analysis. Drug Alcohol Depend 2015;154:1-13.

20. Drake RE, Essock SM, Shaner A, et al. Implementing dual diagnosis services for clients with severe mental illness. Psychiatr Serv 2001;52:469-76.

21. Lagisetty P, Klasa K, Bush C, Heisler M, Chopra V, Bohnert A. Primary care models for treating opioid use disorders: what actually works? A systematic review. PLoS One. 2017;12:e0186315.

22. Korthuis PT, McCarty D, Weimer M, et al. Primary care-based models for the treatment of opioid use disorder: a scoping review. Ann Intern Med 2017; 166:268-78.

23. Cantone RE, Fleishman J, Garvey B, Gideonse N. Interdisciplinary management of opioid use disorder in primary care. Ann Fam Med 2018;16:83.

24. Mission and History. OCHIN. Available from: https:// ochin.org/mission-history/. Accessed November 15, 2018.

25. Centers for Disease Control and Prevention. 2018 Annual Surveillance Report of Drug-Related Risks and Outcomes-United States. Surveillance Special Report. Hyattsville, MD: Centers for Disease Control and Prevention, U.S. Department of Health and Human Services; 2018. Available from: https://www. cdc.gov/drugoverdose/pdf/pubs/2018-cdc-drugsurveillance-report.pdf

26. Becker WC, Sullivan LE, Tetrault JM, Desai RA, Fiellin DA. Non-medical use, abuse and dependence on prescription opioids among U.S. adults: psychiatric, medical and substance use correlates. Drug Alcohol Depend 2008;94:38-47. 